\title{
Double Modulation Scheme for Switching Mixers Controlled by Sigma-Delta Modulators
}

Nielsen, Per Asbeck; Fallesen, Carsten

Published in:

Electronics, Circuits and Systems, 1998 IEEE International Conference on

Link to article, DOI:

10.1109/ICECS.1998.813341

Publication date:

1998

Document Version

Publisher's PDF, also known as Version of record

Link back to DTU Orbit

Citation $(A P A)$ :

Nielsen, P. A., \& Fallesen, C. (1998). Double Modulation Scheme for Switching Mixers Controlled by Sigma-

Delta Modulators. In Electronics, Circuits and Systems, 1998 IEEE International Conference on (Vol. 1, pp. 363366). IEEE. https://doi.org/10.1109/ICECS.1998.813341

\section{General rights}

Copyright and moral rights for the publications made accessible in the public portal are retained by the authors and/or other copyright owners and it is a condition of accessing publications that users recognise and abide by the legal requirements associated with these rights.

- Users may download and print one copy of any publication from the public portal for the purpose of private study or research.

- You may not further distribute the material or use it for any profit-making activity or commercial gain

- You may freely distribute the URL identifying the publication in the public portal 


\title{
Double Modulation Scheme for Switching Mixers Controlled by Sigma-Delta modulators
}

\author{
Per Asbeck Nielsen ${ }^{1,2}$, Carsten Fallesen ${ }^{1,2}$ \\ ' Center for Integrated Electronics \\ Department of Information Technology \\ Technical University of Denmark \\ DK-2800 Lyngby, Denmark \\ ${ }^{2}$ Nokia Mobile Phones A/S, \\ Frederikskaj, DK-1790 Copenhagen V, Denmark \\ Tel.: +45 216156 02, Fax: +4533292001 \\ E-mail: per.nielsen@nmp.nokia.com
}

\begin{abstract}
A new method of modulating a signal, using Sigma Delta modulation in conjunction with switching- and sub-sampling mixers, is presented. Besides the normal functionality provided by the mixer an additional mixing or modulation function can be achieved with a small modification to the existing mixer. This modification can be carried out on a big amount of mixers including the above mentioned. Although the principle was meant to be used to down convert analog signals the principle is general and can be used in digital circuits too. This paper verifies the new mixing scheme and compares it to the traditional mixing scheme. Also the most suitable applications of the different mixing schemes are discussed.
\end{abstract}

\section{Introduction}

The growing demand of low cost personal communications systems has motivated the intense research in the field of wireless integrated RF frontends. Many new obstacles have to be solved to obtain a single solution IC, which leads to new front-end architectures [1],[2],[3],[4]. This paper deals with a modulation scheme, the double modulation scheme, that utilizes that clocked mixers such as the switching mixer and the subsampling mixer can be modified slightly to provide an extra modulation controlled by a 1 bit Sigma Delta modulated signal. The double modulation scheme can be used in different architectures for baseband modulation or channel selection in the receiver part. The modulation applies to both analog and digital signal processing, but the examples in this paper will focus on down-converting analog RF signals.

The text is divided into the following sections. In section 2 the principle of the switching mixer and the subsampling mixer is described. The purpose is to relate the traditional mixers to the new mixing scheme. In section 3 the principle of the new mixing scheme is presented. Section 4 deals with bandpass modulators in the mixing scheme and its limitations. Section 5 concludes this paper.

\section{The conventional downconversion mixer}

The down conversion mixer in the receiver usually has the functionality of a switching mixer. This is the case of the Gilbert type mixer if it is driven by a strong local oscillator (LO) signal. The LO then modulates only the sign of the RF input signal. There are two obvious reasons for driving the Gilbert cell with a strong LO. The noise amplification by the (MOS) transistors is then lower, since the transistors are only saturated for a small period of time, when the sign changes. The other reason is that in deep submicron CMOS processes it is difficult to predict the non linear deviation from the ideal multiplier. In figure 1 two realizations of switching mixers are depicted.

The change of sign is equivalent to a multiplication of
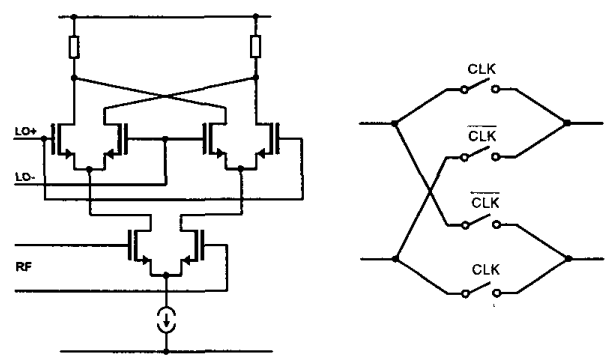

Fig. 1. Switching mixers. Left: Gilbert cell with strong LO signal; Right differential CMOS sign switch.

the RF signal with a square wave signal. The squarewave is expressed by the trigonometric series below. 


$$
\begin{aligned}
s q(t) & =\left\{\begin{array}{cc}
+1 & n T<t \leq(n+0.5) T \\
-1 & \text { otherwise }
\end{array}\right. \\
& =\frac{4}{\pi} \cdot \sum_{n=0}^{\infty} \frac{1}{1+2 n} \cdot \sin \left((1+2 n) \cdot \frac{2 \cdot \pi}{T} \cdot t\right)
\end{aligned}
$$

It can be seen that it contains all harmonics of the fundamental frequency, but they are attenuated with the frequency. If disturbances or noise is present at the harmonic frequencies of the LO signal, they will be attenuated as they translate into the base band.

This is not the case for the subsampling-mixer. All disturbances at the harmonics will be weighted equally in the base band. The principle is shown in fig. 2, where the switches are controlled by a clock signal. At the falling edge of the clock the switches open and the momentary value of the RF signal is sampled and held on the capacitor. This value can then be processed by the following $\mathrm{A} / \mathrm{D}$ converter. The sub-sampling process can be seen as a multiplication of the input signal with an infinite sum of Dirac impulses spaced by the sampling period.

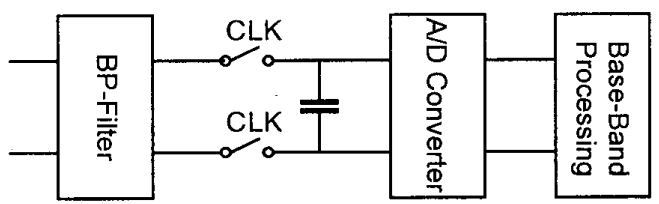

Fig. 2. Principle of a differential realization of subsampling.

The output spectrum of the subsampler can be described as

$$
F_{D r}(f)=\sum_{n=-\infty}^{\infty} F_{C r}\left(f-2 \cdot \pi \cdot f_{s} \cdot n\right)
$$

where $F_{c r}$ is the spectrum of the RF input signal. $F_{D r}$ is the sampled signal and $f_{x}$ is the sampling frequency.

\section{Double modulation scheme using Sigma Delta Modulation}

The double modulation scheme can be used with mixers, where it is possible to modify the mixer so that the sign of the output of the mixer is controlled externally. The sign is controlled by a 1 bit Sigma Delta modulated sine signal. The subsampling mixer in figure 2 can be modified by adding 2 more switches to accommodate the sign control. In figure 3 the double modulation scheme using a subsampling mixer is shown.



Fig. 3.Double demodulation using a subsamplingmixer

The advantage of using a subsampling mixer is that the sampling frequency is much lower than the RF carrier frequency, so the clock signal of the Sigma Delta modulator can be used for the sampling. Typically the sampling frequency is between $10 \mathrm{MHz}$ and $50 \mathrm{MHz}$ [5],[6], [7] which is the operation frequencies that can be achieved in a submicron CMOS process, with switched capacitor circuits or fully digital resonators [8]. The change of sign can be understood as a multiplication of the analog RF signal with a 1 bit signal.

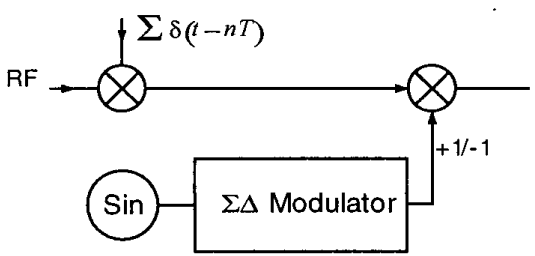

Fig. 4. Block diagram of the modified modulator.

The output of the mixer is therefore the result of folding the subsampled RF signal, with the spectrum of the output of the Sigma Delta modulator.

$$
F_{\text {demod }}(f)=\underbrace{\left(\sum_{n=-\infty}^{\infty} F_{R F}\left(f-2 \cdot \pi \cdot f_{s} \cdot n\right)\right)}_{\text {Subsampled spectrum }} * F_{\Sigma \Delta}(f)
$$

The spectrum of the Sigma Delta modulated signal is a sum of the spectrum of the sine and the typical quantization noise spectrum of Sigma Delta modulator. The power spectral density (PSD) of the noise is approximated with the following function [9] assuming that all zeroes in the noise transfer function are at DC

$\operatorname{PSD}(f)=\frac{2^{2 n}}{12 \cdot f_{s}} \cdot\left(\sin \left(\pi \cdot \frac{f}{f_{s}}\right)\right)^{2 \cdot n} \approx \frac{1}{12 \cdot f_{s}} \cdot\left(2 \pi \cdot \frac{f}{f_{s}}\right)^{2 \cdot n}$

Where $\mathrm{n}$ is the order of the Sigma Delta modulator.

To verify the double modulation scheme a simulation with a Sigma Delta modulated sine signal was used to 
control the sig.t of the sampled RF signal. In figure 5 the spectrum of the sign control signal, with a peak at the frequency $0.012 f$, and the RF signal with peaks at $0.016 f_{s}$ and $0.020 f_{s}$ are depicted. One can see the typical quantization noise, increasing with the frequency, originating from the Sigma Delta modulator.

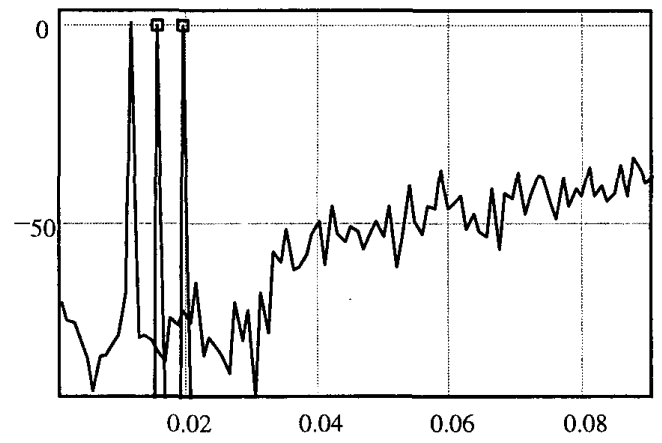

Fig. 5 Sigma Delta modulated switch steering signal and the sampled RF marked with 2 boxes.

The output of the mixer is shown in figure 6 . The spectrum of the up and down converted RF signal and the original RF spectrum can be seen. The frequency translation acts exactly like an ideal multiplier, except for the quantization noise of the Sigma Delta modulator. For a given PSNR the bandwidth is limited by quantization noise of the Sigma Delta modulator.

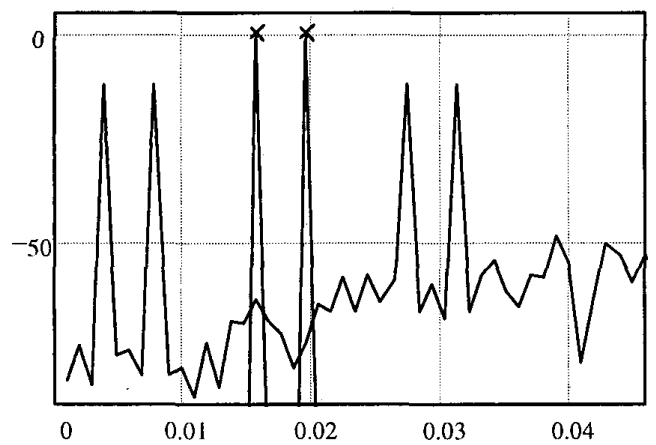

Fig. 6 Out of the mixer, up and down converted RF signal, and .RF input (marked with crosses).

As mentioned in the beginning the double modulation can also be achieved with a Gilbert mixer. The "change sign" unit should in this case be inserted between the LO and the LO input (the two upper differential pairs) of the Gilbert cell. In this way one is able to modulate the LO with a Sigma Delta modulated sine signal and obtain a frequency shift of the LO before it is lead into the Gilbert cell. The advantage of this configuration is that the RF signal path is fully time continuous and no outof-band noise is folded into the baseband as is the case with a subsampling mixer. It is therefore possible to filter out the out-of-band noise after the mixing.

\section{Bandpass Demodulation}

In some cases it is a limiting factor that the down sampled RF signal (see figure 3) must be between $0 \mathrm{~Hz}$ and $\mathrm{f} / \mathrm{OSR}$ to avoid the quantization noise. OSR is the over-sampling ratio. In some cases it is convenient if a band starting higher than $0 \mathrm{~Hz}$ can be selected. This is possible if a Bandpass Sigma Delta modulator is used. However, there is a constraint that can be seen in the simulations shown in figure 7 and figure 8 . Figure 8 shows the output of the mixer in two situations. In the

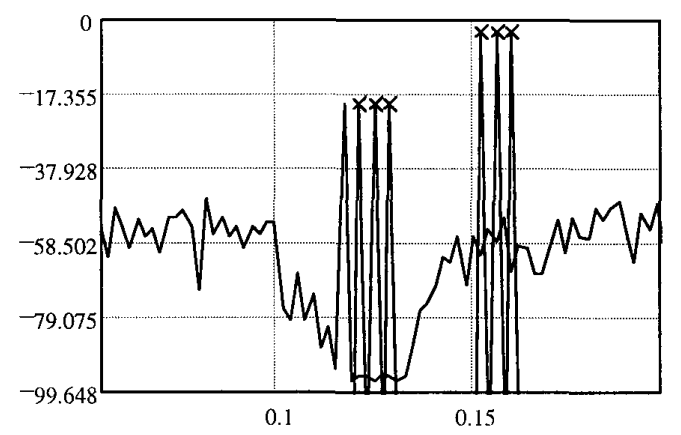

Fig. 7 Out of the mixer, up and down converted RF signal, and $\mathrm{RF}$ input (marked with crosses).

first situation, the whole down-sampled RF band is within the noise shape band of the Sigma Delta

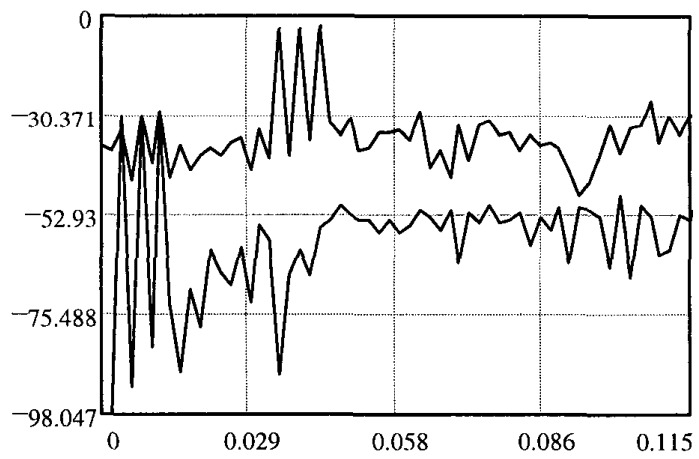

Fig. 8 Out of the mixer, up and down converted RF signal, and.$R F$ input (marked with crosses).

modulator (lower graph). In the other situation some of the spectral components lies outside the noise shape band of the Sigma Delta modulator. These components could represent neighbor channels or disturbers.

In the case of no spectral component outside the noise shape band the double demodulation translates the RF signal to the baseband with high PSNR (lower graph in figure 7). The distance between the signal peak and the noise floor is $60 \mathrm{~dB}$. In the case of spectral components 
outside the noise shape band, the distance is diminishing (upper graph). This is because the spectral components outside the noise shape band down converts the massive quantization noise to the baseband and thereby destroys the PSNR. The sampled RF signal is shown (with crosses in figure 7) together with the sign control signal to the switches. There are three RF spectral components within the noiseshape band ( $\mathrm{f}=0,121 f_{s}, 0.125 f_{s}$, $\left.0.129 f_{s}\right)$ and three outside $\left(\mathbf{f}=0.152 f_{s}, 0.156 f_{s}\right.$, $0.160 f_{s}$ ). The spectral components of the RF signal outside the noise shape band are $20 \mathrm{~dB}$ stronger to emphasize the destructive effect.

It is clearly important that the noise shape band covers the whole down-sampled RF band, in order to maintain a high PSNR.

\section{Conclusion}

A new modulation scheme that introduces two modulations in one mixer, here called the double modulation scheme, was presented. With minor change to existing subsampling-mixers and switching-mixers a second modulation is introduced, by modulating the sign of the analog RF signal in the mixer. Controlling the sign with a 1 bit Sigma Delta modulator, it was shown that an extra additional frequency translation was introduced. Equations describing the noise and the translation of the RF signal were given. Simulations verifying the principles were presented.

\section{References}

[1] "Low-Power Radio-Frequency IC's for Portable Communications", Asad A. Abidi, UCLA, Proc. of the IEEE, vol. 83, no. 4, 1995, pp. 544-569.

[2] "A $1 \mathrm{GHz}$ CMOS RF Front-End IC for a DirectConversion Wireless Receiver", Ahmadreza Rofougaran, James Y.-C. Chang, Maryam Rofougaran, A.A. Abidi, IEEE J. of Solid-State Circuits,Vol. 31, No. 7, July 1996 , pp.880-889

[3] "A Single-Chip $900 \mathrm{MHz}$ CMOS Receiver Front-End with a High Performance Low-IF Topology", Jan Crols, Michiel S. J. Steyaert, IEEE J. of Solid-State Circuits, Vol. 30, No. 12, Dec. 1995, pp.1483-1492.

[4] Jacques C. Rudell, Jia-Jiunn Ou, Thomas Byunghak Cho, George Chien, Francesco Brianti, Jeffrey A. Weldon, Paul R. Gray, "A 1.9 Wide-Band IF Double Conversion CMOS Receiver for Cordless Telephone Applications", IEEE J. of Solid-State Circuits, Vol. 32, No. 12, pp. 2071-2087, Dec. 1997.

[5] "A Highly Linear 1-GHz CMOS Downconversion Mixer", P.Y. Chan, A. Rofougaran, K. A. Ahmed, A.A. Abidi, Proc. European Solid-State Circuits Conference, 1993, Spain, pp. 210-213.

[6] "A Fully Differential $2 \mathrm{GHz}$ CMOS Subsampling-Mixer with SC-Amplifier", Per Asbeck Nielsen, Carsten Fallesen, Allan Jørgensen, Proc. $15^{\text {th }}$ Norchip Conference, Tallinn, Lithuania, 1997

[7] "A CMOS 1.8GHz Sub-sampling Down-Converter for Digital Receiver",Saska Lindfors, Jarkko Jussila, Mika
Länsirinne, Petteri Litmanen, Kari Halonen, Mixed Design of Integrated Circuits and Systems (MIXDES), Education of Computer Aided Design of Modern Devices an ICs, Poznan, Poland, 12-14 June, 1997

[8] "High Frequency Sinusidial Generation Using DeltaSigma Modulation Techniques",Benoît R. Viellette, Gordon W. Roberts, Proc. International Symposium on Circuits and Systems 1995.

[9] "An Overview of Sigma_Delta Converters", Pervez M. Aziz, Henrik V. Sorensen, Jan Van der Spiegel, IEEE Signal Processing Magazine, Jan. 1996

[10]"A 74dB Dynamic Range, 1.1-MHz Signal Band 4th-Order 2-1-1 Cascade Multi-Bit CMOS $\Sigma \Delta$ Modulartor for ADSL", F. Medeiro, B. Perez-Verdu, A. RodriguezVazquez, Proc. 23th European Solid-State Circuits Conference, Southampton, UK, 16-18 September. 